\title{
Diffusive lossless energy and coherence transfer by noisy coupling
}

\author{
D. Mogilevtsev ${ }^{1}$, G. Ya. Slepyan ${ }^{2}$ \\ ${ }^{1}$ Institute of Physics, Belarus National Academy of Sciences, F.Skarina Ave. 68, Minsk 220072 Belarus; \\ ${ }^{2}$ Department of Physical Electronics, School of Electrical Engineering, \\ Faculty of Engineering, Tel Aviv University, Tel Aviv 69978, Israel
}

\begin{abstract}
Here we show that noisy coupling can lead to diffusive lossless energy transfer between individual quantum systems retaining a quantum character leading to entangled stationary states. Coherence might flow diffusively while being summarily preserved even when energy exchange is absent. Diffusive dynamics persists even in the case when additional noise suppresses all the unitary excitation exchange: arbitrarily strong local dephasing, while destroying quantum correlations, is not affecting energy transfer.
\end{abstract}

PACS numbers: 03.65.Yz,05.70.-a,05.40.Ca

Introduction Diffusive transfer of energy (and, ultimately, derivation of Fourier heat-transfer law from microscopic dynamics) up to day remain subjects of theoretical interest and even controversy [1 5]. For microscopic dynamics dominated by quantum effects establishing of diffusive energy transfer is far from being obvious. Commonly considered microscopic models, such as chains of unitarily connected networks of bosonic and/or fermionic systems with attached thermal reservoirs and noise sources can demonstrate both ballistic and diffusive behavior in dependence on interaction strengths and other parameters of the whole system, generally requiring approximations (such as long time and large size limits) for emergence of classical-like heat dynamics. Here we suggest a noise-mediated microscopic mechanism for diffusive transfer. Energy can propagate without loss, but through losses. Recently it has become quite usual to see noises not only as something destroying quantum coherence and reducing quantum states to classicality, but also as a tool to create and enhance quantumness. Non-local loss can preserve entanglement and even generate entangled stated from initially uncorrelated ones [6]10]. Engineered loss can lead to dissipative protection and coherence preservation [11 13 and deterministic creation of non-classical states [14, 17, 20 and serve as a tool for quantum computing [15, 16]. Networks of dissipatively coupled systems can support topologically protected states [21. Even a pure local dephasing is no longer considered completely harmful: it can enhance quantum state transfer and suppress localizing effects of static disorder 22 24]. However, too strong local dephasing generally suppresses unitary excitation exchange and energy transfer stemming from it.

Here we show a microscopic mechanism of diffusive lossless energy transfer, which is impervious to local dephasing. It arises when coupling constants describing common single-excitation hopping are fluctuating randomly, like it is, for example, with spin-spin dipolar coupling in random environments. Dynamics produced by fluctuating coupling might preserve certain quantum correlations, and even entanglement during evolution toward the stationary state. Populations are not coupled by the dynamics with the off-diagonal terms. So, for example, a diagonal initial state evolves to a symmetrical mixture of diagonal states, whereas coherences (i.e., off-diagonal elements or superpositions of them) can also flow diffusively, and the sum of certain coherences can be preserved by dynamics. Diffusive coherence flow can occur without energy exchange. Such a dynamics can occur for different quantum systems, for example, two-level systems and bosonic modes. The latter case is remarkable. It is known that the light in a structured surrounding (such as photonic crystals) can produce coherent dynamics typical rather for charged or neutral particles, but not for photons, such as Blokh oscillations with single photons in the waveguide lattices [25] 27, Rabi-oscillations of photons [28 and effective magnetic field for photons by controlling the phase of dynamic modulation 29. Here we have one more effect: photon diffusion without energy losses due to noisy coupling.

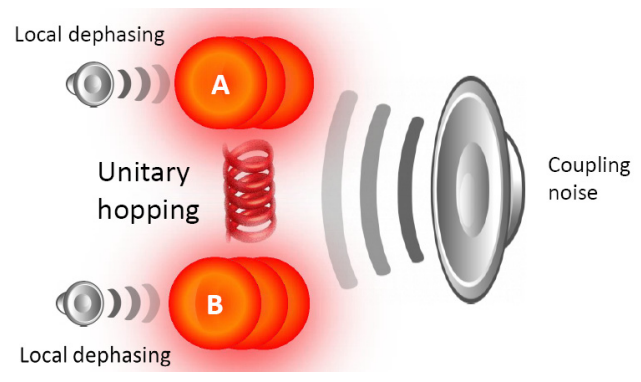

FIG. 1: (color online) A schematic depiction of the considered scheme with possible local dephasing noises affecting transition frequencies, possible non-fluctuating unitary hopping and non-local coupling noise (described by variables $\eta_{j}(t)$ in Eq.(1)) affecting both unitary hopping rate and transition frequencies.

Simple model To show the essence of our diffusive transfer mechanism, let us start with the simple illustrative model. We consider a tight-binding chain of identical two-level systems (TLS) with the following interaction Hamiltonian

$$
H=\hbar \sum_{j=1}^{N} \eta_{j}(t)\left(\sigma_{j}^{+}+\sigma_{j+1}^{+}\right)\left(\sigma_{j}^{-}+\sigma_{j+1}^{-}\right),
$$


where the operators $\sigma_{j}^{ \pm}=\left| \pm_{j}\right\rangle\left\langle\mp_{j}\right|$; the vector $\left| \pm_{j}\right\rangle$ describes the upper (+) or lower (-) levels of $j$-th TLS (see Fig 11. Quantities $\eta_{j}(t)$ describe classical real zero-mean independent white noises, $\left\langle\eta_{j}(t)\right\rangle=0,\left\langle\eta_{j}(t) \eta_{k}(\tau)\right\rangle=$ $\gamma_{j} \delta_{j k} \delta(t-\tau)$; quantities $\gamma_{j} \geq 0, \forall j$ we term "hopping diffusion rates". Physically, the model (1) corresponds to the chain with the same noise affecting only two neighbors with the energy levels and interaction strengths perturbed in the same way 30. Deriving the master equation in the standard way [31, one gets from Eq.(1) the following master equation

$$
\frac{d}{d t} \rho=2 \sum_{j} \gamma_{j}\left(L_{j} \rho L_{j}-L_{j} \rho-\rho L_{j}\right)
$$

with $L_{j}=\left(\sigma_{j}^{+}+\sigma_{j+1}^{+}\right)\left(\sigma_{j}^{-}+\sigma_{j+1}^{-}\right)$. It is obvious from Eq. (2) that the sum of TLS upper-level populations (which we further address as energy), $E_{0}=\sum_{j=1}^{N+1} n_{j}$, where $n_{j}=\left\langle\sigma_{j}^{+} \sigma_{j}^{-}\right\rangle$, is preserved. Eq. 2 leads directly to the diffusive transfer equation for individual populations

$$
\frac{d}{d t} n_{j}=-2\left(\gamma_{j}+\gamma_{j-1}\right) n_{j}+2 \gamma_{j} n_{j+1}+2 \gamma_{j-1} n_{j-1}
$$

Further, assuming the chain homogeneous, we take $\gamma_{j} \equiv$ $\gamma$ for $1 \leq j \leq N$ and $\gamma_{j}=0$ for $j \leq 0, j \geq N+1$. it follows from Eq. (3) that in the long time limit the equilibrium is reached, $n_{j} \rightarrow n_{s t}=E_{0} /(N+1), \forall j$. Introducing a sum of local energies from $k$ th to $l$ th TLS, $E_{k, l}(t)=\sum_{j=k}^{l} n_{j}(t)$, $k, l \neq 1, N+1$, from Eq. $[3]$ it follows that $\frac{d}{d t} E_{k, l}=S_{l+1}-$ $S_{k-1}$, with the local flux defined as $S_{k}=2\left(n_{k+1}-n_{k}\right)$. The energy balance in a region of the chain is naturally defined by the energy flowing through the borders.

(a)

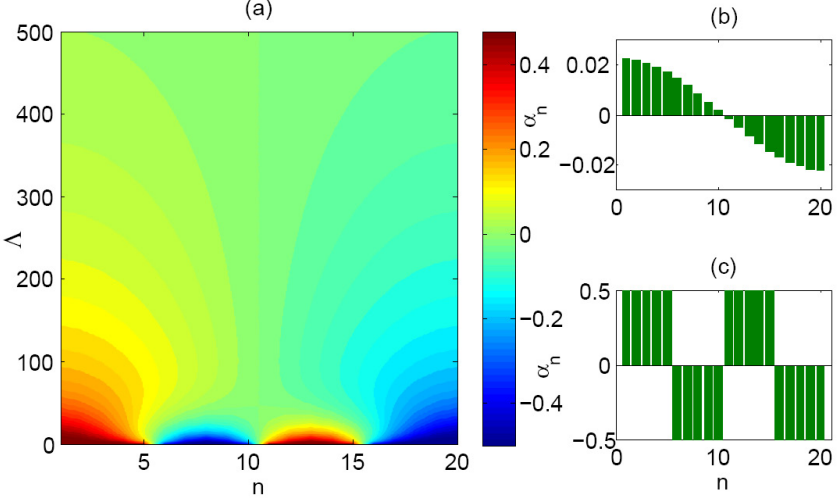

FIG. 2: (color online)(a)Dynamics of coherences, $\alpha_{n}$, as given by Eq. (2) for the energy-equilibrated initial state (4) with initial distribution of $\alpha_{n}$ shown in the panel (c). The panel (b) shows distribution of $\alpha_{n}$ for $\Lambda=500, \Lambda=\gamma t$.

Non-classicality Diffusive energy transfer does not imply loss of quantum correlations of the chain state. Let us demonstrate it with an example of singleexcitation dynamics. Firstly, the system described by Eq. 22 has an entangled stationary state $\left|\psi_{s t}\right\rangle=$ $\sum_{j=1}^{N+1}(-1)^{j}\left|1_{j}\right\rangle / \sqrt{N+1}$, satisfying $L_{j}\left|\psi_{s t}\right\rangle=0, \forall j$, where the vector $\left|1_{j}\right\rangle=\left|+{ }_{j}\right\rangle \prod_{k \neq j}\left|-{ }_{k}\right\rangle$. Similar entangled stationary states were found recently in dissipatively coupled TLS chains [32]. Secondly, coherences in the chain can also flow diffusively having the sum of them preserved. For example, assuming the single-excitation initial state, for coherences defined as, $\alpha_{k}=(-1)^{k}\left\langle 1_{k}|\rho| 0\right\rangle$, one has equation formally coinciding with the equation for a classical random walk

$$
\frac{d}{d t} \alpha_{k}=2 \gamma\left(-2 \alpha_{k}+\alpha_{k+1}+\alpha_{k-1}\right)
$$

for $1<k<N+1$, where $|0\rangle$ is the vector describing all the TLS of the chain being in the lower level. Curiously, the coherence might flow even if the energy gradient is absent. Indeed, this will take place, for example, for the initial state being a mixture of phase states of each TLS:

$$
\rho(0)=\sum_{j=1}^{N+1}\left|\theta_{j}\right\rangle\left\langle\theta_{j}\right| /(N+1),
$$

where $\left|\theta_{j}\right\rangle=\left(\left|+{ }_{j}\right\rangle+\exp i \theta_{j}\left|-{ }_{j}\right\rangle\right) \prod_{k \neq j} \mid-{ }_{k}>/ \sqrt{2}$, and the angles $\theta_{j}$ are arbitrary. In the long-time limit the state will be the following mixture $\rho(\infty)=\sum_{j=1}^{N+1} \rho_{j}$, where $\rho_{j}=\left(\mathbf{1}_{j}+(-1)^{j}\left(\Theta^{*} \sigma_{j}^{+}+\Theta \sigma_{j}^{-}\right)\right) \otimes \prod_{k \neq j}\left|-{ }_{k}\right\rangle\left\langle-{ }_{k}\right| / 2(N+1)$, where $\Theta=\sum_{j=1}^{N+1}(-1)^{j} \exp \left\{i \theta_{j}\right\}$. This situation is illustrated in Fig 2, where it is shown how evolves the state (4) with $\theta_{j}$ equal to 0 or $\pi$. Coherences, $\alpha_{n}$, oscillate between positive and negative values. However, energy exchange is not taking place at all times. For this example $\Theta=0$, so, asymptotically the state of the chain is the diagonal mixture of the vacuum and single-excitation states. For long times, $4 \gamma t \sin ^{2}\{\pi /(N+1)\} \gg 1$, phase of chain halves are opposite, since the initial state depicted in Fig 2(c) has non-zero overlap with the simplest antisymmetric eigenmode of the chain 32. For coherences $\alpha_{k l}=(-1)^{k+l}\left\langle 1_{k}|\rho| 1_{l}\right\rangle$ and $k \neq l \pm 1$ Eq. 2 for $1<k, l<N+1$ one obtains equations for $2 \mathrm{D}$ classical random walk:

$$
\frac{d}{2 \gamma d t} \alpha_{k l}=-4 \alpha_{k l}+\alpha_{k+1, l}+\alpha_{k-1, l}+\alpha_{k, l+1}+\alpha_{k, l-1} .
$$

For $k=l \pm 1$ one has equations not coinciding with ones for $2 \mathrm{D}$ classical random walk

$$
\frac{d}{2 \gamma d t} \alpha_{k, k+1}=-2 \alpha_{k, k+1}+\alpha_{k-1, k+1}+\alpha_{k, k+2} .
$$

It is seen from these equations that quantities $\alpha_{k l}$ for $k \neq$ $l$ are not coupled with populations, $\rho_{k k}$. However, they 
are not arbitrary. The matrix $\rho(t)$ should always be semipositive, so, for example, one has $\left|\alpha_{k l}(t)\right| \leq \sqrt{\rho_{k k}(t) \rho_{l l}(t)}$ for any $t$. Absence of coupling between diagonal and offdiagonal elements of the density matrix leads to preservation of the diagonality of the state.

Symmetrization As follows from Eq.(2), a single initial excitation leads asymptotically to the symmetrical mixture $\rho(\infty)=\sum_{j=1}^{N+1}\left|1_{j}\right\rangle\left\langle 1_{j}\right| /(N+1)$. Now let us demonstrate that the symmetrization takes place for an arbitrary initial diagonal state. Indeed, for the variable $n(\mathcal{K})=\left\langle\sigma_{j_{1}}^{+}(t) \sigma_{j_{1}}^{-}(t) \ldots \sigma_{j_{K}}^{+}(t) \sigma_{j_{K}}^{-}(t)\right\rangle$, where $\forall j_{k} \in \mathcal{K}$ one has from Eq. 22 that

$$
\begin{aligned}
\frac{d}{d t} n(\mathcal{K})= & -2 \sum_{j \in \mathcal{K}} \gamma_{j} \varsigma_{j+1}(n(\mathcal{K})-n(\mathcal{K}: j \rightarrow j+1))- \\
& -2 \sum_{j \in \mathcal{K}} \gamma_{j-1} \varsigma_{j-1}(n(\mathcal{K})-n(\mathcal{K}: j \rightarrow j-1)),(5)
\end{aligned}
$$

where the set $(\mathcal{K}: j \rightarrow k)$ is the set $\mathcal{K}$ with $j$-th TLS replaced with $k$-th; the coefficients $\varsigma_{k}=0$ for $k \in \mathcal{K}$ and are unity otherwise. Eq. (5) leads to a number of interesting consequences. For examples, not only excitations, but also the absence of excitations can propagate diffusively. Let us consider vectors $\left|0_{j}\right\rangle=\left|-_{j}\right\rangle \prod_{k \neq j}\left|+_{k}\right\rangle$. It follows from Eq. (5) that the probability, $n_{j}=\left\langle 0_{j}|\rho| 0_{j}\right\rangle$ of non-excitation of the chain TLS satisfies the 1D diffusion equation (3). Just like it was for the single-excitation case, off-diagonal elements do not couple to the diagonal ones. But the most importantly, the diffusion equation (5) shows that any initial diagonal state $\rho(0)=\sum_{n=0}^{N+1} \rho_{n}$, with energy $E=\sum_{n=0}^{N+1} \operatorname{Tr}\left\{\rho_{n}\right\} n$, where $\rho_{n}$ are states with exactly $n$ excited TLS, is transformed to the symmetrical state

$$
\rho(0) \rightarrow \rho(\infty)=\sum_{n=0}^{N+1} \operatorname{Tr}\left\{\rho_{n}\right\} S_{n, N+1},
$$

which the same energy, where $S_{n, N+1}$ is the phaseaveraged Dicke state being an equal mixture of projectors of all permutations of $n$ excited TLS from the total number, $N+1$, of them 33 .

Thermalization Dynamics described by Eqs. (35) preserves the number of excitation and does not lead to the global Gibbs state of the chain. However, local populations, $n_{j}$, still can be given a meaning of the local temperature at least for states close to the stationary one (6). Firstly, any single-particle state obtained by averaging over $N$ TLS of the state (6), is the Gibbs state, $\rho_{j}(\infty)=$ $\exp \left\{-\beta \sigma_{j}^{+} \sigma_{j}^{-}\right\} / \operatorname{Tr}\left(\exp \left\{-\beta \sigma_{j}^{+} \sigma_{j}^{-}\right\}\right)$: where the parameter $\beta=-\ln \{(1-\bar{n}) / \bar{n}\}$, and $\bar{n}=\sum_{n=0}^{N+1} \operatorname{Tr}\left\{\rho_{n}\right\} n /(N+1)$ being the population of each TLS. Then, the state $(6)$ is the typical one for which the canonical typicality takes place [34, 35]. Namely, for small subset of $m \ll N+1$ TLS the state of these $m$ TLS averaged over the rest of the chain, is very close to the to the Gibbs state $\rho_{m}^{\text {Gibbs }}=$ $\exp \left\{-\beta H_{m}\right\} / \operatorname{Tr}\left(\exp \left\{-\beta H_{m}\right\}\right)$ with $H_{m}$ is the sum of $\sigma_{j}^{+} \sigma_{j}^{-}$of the subset. For TLS and mixed state (6) there is the exact bound, $\left\|\rho_{m}(\infty)-\rho_{m}^{\text {Gibbs }}\right\|_{1} \leq 4 m /(N+1)$, where $\rho_{m}(\infty)$ is the state of the subset with $m$ TLS averaged over the rest of the chain [36]. Thus, for the state of the chain weakly deviating from the symmetric one (6), one can meaningfully introduce the temperature as $T=\hbar \omega / \mathrm{k} \beta$, and derive the continuous heat-transfer equation. Let us do it as outlined in Ref. 37]. For the heat flux between the neighbor TLS in the continuous limit one has $J=2 \gamma_{j} \hbar \omega\left(n_{j+1}-n_{j}\right) \rightarrow-2 C_{V}(T) a \gamma(x) \frac{\partial}{\partial x} T$, where $a$ is the distance between the neighbor TLS, and the specific heat $C_{V}=\partial u / \partial T$, and the local internal energy is $u(x)=\hbar \omega n(T(x))$. Thus, one gets the following Fourier equation

$$
\frac{\partial}{\partial t} u \approx-\frac{\partial}{\partial x}\left(\kappa(x, T) \frac{\partial}{\partial x} T\right)
$$

where the thermal conductivity $\kappa(x, T)=$ $2 a^{2} \gamma(x) C_{V}(T)$. The specific heat for the chain is $C_{V}=\mathrm{k} \beta^{2} e^{\beta}\left(e^{\beta}+1\right)^{-2}$. The dependence of the heat conductivity of the temperature is defined by the hopping diffusion rate, $\gamma$. For the rate independent of the chain temperature, one has a common $T^{-2}$ dependence 38.

Generalizations and realizations Emergence of the diffusive lossless energy transfer through coupling noise is quite general phenomenon not restricted to TLS systems. For a single-excitation case the dynamics is completely similar for TLS chain or bosonic one, or even the system of coupled TLS and modes. Obviously, any unitary hopping term preserving the number of excitations and with zero-mean random interaction constants under condition of the Markovian approximation applicability would lead to diffusive energy transfer. For the chain of bosonic modes the hopping term is

$$
H=\hbar \sum_{j=1}^{N} \eta_{j}(t)\left(a_{j}^{+}+a_{j+1}^{+}\right)\left(a_{j}+a_{j+1}\right),
$$

with bosonic creation, $a_{j}^{+}$, and annihilation, $a_{j}$ operators satisfying $\left[a_{j}, a_{k}^{+}\right]=\delta_{j k}$. It is easy to see that for the independent white noises, $\eta_{j}(t)$, one gets the standard Lindblad master equation with $L_{j}=\left(a_{j}^{+}+\right.$ $\left.a_{j+1}^{+}\right)\left(a_{j}+a_{j+1}\right)$, which leads directly to the diffusive transfer equation (3) for the modal average number of photons, $n_{j}=\left\langle a_{j}^{+} a_{j}\right\rangle$. Just like the TLS chain, diagonal and off-diagonal elements of the total chain density matrix in the energy basis are not coupled by dynamics. Also, the initial diagonal states remain diagonal and are asymptotically symmetrized.

Bosonic scheme offers the simplest way to realize, test and use the discussed diffusive transfer scheme. For example, the system of coupled waveguides similar to ones recently used for demonstration of localized states in ideal defectless Lieb lattices can be used for a purpose [39, 40. Modulation of waveguide coupling constants 
achieved by random variation of distance, waveguide dimension and/or dielectric constant of the bulk can be implemented. In this way, it is possible realizing a lossless optical equalizer for suppressing both intensity and phase fluctuations of multi-mode fields, which is currently a topical problem [41] Another possible realizations one can find in schemes for dynamical suppression of decoherence [42, in arrays of persistent-current Josephson qubits [43] with dynamical coupling [44, or the chain of nitrogenvacancy centers in diamond in fluctuating magnetic field [45.

Local dephasing and unitary hopping In any realistic schemes involving random uncontrolled variation of coupling constants, one naturally expects having local dephasing. Also, when fluctuating coupling constants are not of zero mean, one should expect unitary hopping. To consider an influence of these phenomena on the energy transfer, let us again consider the chain of TLS. We take the interaction Hamiltonian as

$$
\begin{gathered}
\frac{H}{\hbar}=\sum_{j=1}^{N+1} R_{j}(t) \sigma_{j}^{+} \sigma_{j}^{-}+\sum_{j=1}^{N} G_{j}(t)\left(\sigma_{j}^{+} \sigma_{j}^{-}+\sigma_{j+1}^{+} \sigma_{j+1}^{-}\right)+ \\
\sum_{j=1}^{N}\left(\left(g_{j}+v_{j} G_{j}(t)\right) \sigma_{j}^{+} \sigma_{j+1}^{-}+\left(g_{j}^{*}+v_{j}^{*} G_{j}(t)\right) \sigma_{j+1}^{+} \sigma_{j}^{-}\right),
\end{gathered}
$$

where constants $g_{j}$ describe the excitation exchange strengths between the neighbor TLS; the constants $v_{j}$ describe strengths of TLS interaction with the corresponding reservoir. Operators $R_{j}(t)$ are describing local dephasing; operators $G_{j}(t)$ describe common reservoirs for neighbor TLS. We assume the Markovian limit taking $\left\langle X_{j}(t) Y_{k}(\tau)\right\rangle=\gamma_{j}^{X, Y} \delta_{X Y} \delta_{j k} \delta(t-\tau)$ with $X, Y=R, G$. Again, the standard derivation procedure leads from the Hamiltonian (9) to the following master equation

$$
\begin{array}{r}
\frac{d}{d t} \rho=i[V, \rho]+\sum_{j=1}^{N+1} \gamma_{j}^{R}\left(2 L_{j}^{R} \rho L_{j}^{R}-L_{j}^{R} \rho-\rho L_{j}^{R}\right)+ \\
\sum_{j=1}^{N} \gamma_{j}^{G}\left(2 L_{j}^{G} \rho L_{j}^{G}-\left(L_{j}^{G}\right)^{2} \rho-\rho\left(L_{j}^{G}\right)^{2}\right),
\end{array}
$$

where $L_{j}^{R}=\sigma_{j}^{+} \sigma_{j}^{-}, L_{j}^{G}=\sigma_{j}^{+} \sigma_{j}^{-}+\sigma_{j+1}^{+} \sigma_{j+1}^{-}+v_{j} \sigma_{j}^{+} \sigma_{j+1}^{-}+$ $v_{j}^{*} \sigma_{j+1}^{+} \sigma_{j}^{-}$, and the unitary part is $V=\sum_{j=1}^{N}\left(g_{j} \sigma_{j}^{+} \sigma_{j+1}^{-}+\right.$ $\left.g_{j}^{*} \sigma_{j+1}^{+} \sigma_{j}^{-}\right)$. Deriving equations for average populations, from Eq. 10 one gets

$$
\begin{array}{r}
\frac{d}{d t} n_{j}=i \sum_{k=j-1, j}\left\langle g_{k} \sigma_{k}^{+} \sigma_{k+1}^{-}-g_{k}^{*} \sigma_{k+1}^{+} \sigma_{k}^{-}\right\rangle+ \\
2\left(\gamma_{j}\left|v_{j}\right|^{2}+\gamma_{j-1}\left|v_{j-1}\right|^{2}\right) n_{j}- \\
2 \gamma_{j}\left|v_{j}\right|^{2} n_{j+1}-2 \gamma_{j-1}\left|v_{j-1}\right|^{2} n_{j-1}
\end{array}
$$

It is immediately seen from Eq. (11) that in absence of unitary exchange, $g_{k}=0, \forall j$, localized dephasing does not influence at all the diffusive transfer caused by fluctuating coupling constants. The unitary hopping is more harmful, since it couples diagonal and off-diagonal matrix elements. However, in this case local dephasing can actually save the day. Let us assume that localized dephasing is by far the strongest factor influencing dynamics of TLS (as it natural for realistic noisy structures and larger temperatures, when the Markovian limit holds for dephasing [31]), $\gamma_{j}^{R} \gg \gamma_{k}^{R},\left|v_{k}\right|^{2} \gamma_{k}^{R},\left|g_{k}\right|, \forall j, k$. Then, it is easy to get from Eq. 111 that $\left\langle\sigma_{k}^{+}(t) \sigma_{k+1}^{-}(t)\right\rangle \approx$ $\left\langle\sigma_{k}^{+}(0) \sigma_{k+1}^{-}(0)\right\rangle \exp \left\{-\left(\gamma_{k}^{R}+\gamma_{k+1}^{R}\right) t\right\}+O\left(\left[\min _{j} \gamma_{j}^{R}\right]^{-1}\right)$. Strong local dephasing suppresses unitary excitation exchange. However, this dephasing does not affect energy transfer produced by the correlated dephasing. Thus, for times much exceeding $\max \left\{1 / \gamma_{j}^{R}\right\}$ and hopping diffusion rates much exceeding $\max \left\{\left|g_{j}\right|^{2} / \gamma_{j}^{R}\right\}$ the first term on the right-hand part of Eq. (11) can be neglected, and populations, $n_{j}(t)$ change diffusively as described by Eq.(3). Due to possibility of neglecting the unitary hopping, also correlation functions $n(\mathcal{K})$ would evolve according to Eq.(5) with the final state being symmetrized and thermalized, as it was described by Eq. (6).

Conclusions We have discussed microscopic mechanism that leads to diffusive lossless energy transfer on the level of few quanta. We have shown that noise of unitary hopping constants under the conditions of the Markovian approximation applicability leads to the energy diffusion in a tight-binding systems of quantum systems of different nature, be it, for example, spins or field modes. In absence of additional local dephasing, the stationary state can be entangled. Local dephasing, even strong to such extent that unitary hopping is suppressed, does not break the dynamics leading to complete symmetrization of the stationary state. For sufficiently large systems, locally such a stationary state is very close to the Gibbs state. Thus, one can introduce a temperature in a standard way and derive the heat-transfer equation. The dynamics was considered for 1D chain, but there are obvious generalizations for 2D and 3D ones. We have suggested practical systems were the scheme can be verified experimentally: the sets of coupled waveguides with fluctuating coupling, dynamically controlled superconducting qubits, color centers in diamonds. The suggested mechanism of diffusive lossless energy transfer can be responsible for energy transfer in strongly noised coupled quantum systems (such as biomolecules) at high temperature.

D.M. thankfully acknowledges the support of the European Commission through the SUPERTWIN project, id.686731, G. Ya. acknoledges the supports by the EU Horizon 2020 project H2020-MSCA-RISE-2014-644076 CoExAN and EU FP7 projects FP7-PEOPLE-2009IRSES-247007 CACOMEL, FP7-PEOPLE-2009-IRSES246784 SPINMET, FP7-PEOPLE-2012-IRSES-316432 QOCaN and FP7-PEOPLE-2013-IRSES-612285 CANTOR. D.M. and G. Ya. thanks A.P. Nizovtsev for fruitful discussions and pointing to relevant references. 
[1] S. Lepri, R.Livi, A. Politi, Phys. Rep. 377, 1 (2003).

[2] J. Gemmer, M. Michel and G. Mahler, Quantum Thermodynamics, Lect. Notes. Phys. 784 (Springer, 2009).

[3] R. Kosloff, Quantum thermodynamics: A dynamical viewpoint, Entropy, 152100 (2013).

[4] F. Bonetto, J. L. Lebowitz, and L. Rey-Bellet, Fourier Law: A challenge to Theorists, In: Mathematical Physics 2000, Imp. Coll. Press, London 2000, pp. 128150.

[5] A. Dhar, Advances in Physics, 57, 457 (2008).

[6] G.M. Palma, K.-A. Suominen and A.K. Ekert, Proc. Roy. Soc. London Ser. A452 567 (1996).

[7] P. Zanardi and M. Rasetti. Noiseless Quantum Codes. Phys. Rev. Lett. 793306 (1997).

[8] D. Braun, Phys. Rev. Lett. 89, 277901 (2002).

[9] F. Benatti, R. Floreanini, M. Piani, Phys. Rev. Lett. 91, 070402 (2003).

[10] D. A. Lidar, K. B. Whaley, Irreversible Quantum Dynamics, Lecture Notes in Physics 622, 83 (2003).

[11] A. R. R. Carvalho, P. Milman, R. L. de Matos Filho, and L. Davidovich, Phys. Rev. Lett. 86, 4988 (2001).

[12] Z.-X. Man, Y.-J. Xia, and R. Lo Franco, Sci. Rep. 5, $13843(2015)$.

[13] Z.-X. Man, Y.-J. Xia, and R. Lo Franco, Phys. Rev. A 92, 012315 (2015).

[14] J. F. Poyatos, J. I. Cirac and P. Zoller, Phys. Rev. Lett. 77, 4728 (1996).

[15] F. Verstraete, M. M. Wolf, and J. Ignacio Cirac, Nature Physics 5, 633 (2009).

[16] F. Pastawski, L. Clemente and J. I. Cirac, Phys. Rev. A 83 012304(2011).

[17] H. Ezaki, E. Hanamura, and Y. Yamamoto, Phys. Rev. Lett. 83, 3558 (1999).

[18] M. Alexanian, S. K. Bose, Phys. Rev. Lett. 85, 1136 (2000).

[19] H. Ezaki, E. Hanamura, and Y. Yamamoto, Phys. Rev. Lett. 85, 1137 (2000).

[20] S. Clark, A. Peng, M. Gu and S. Parkins, Phys. Rev. Lett. 91177901 (2003).

[21] S. Diehl, E. Rico, M. A. Baranov and P. Zoller, Nature Physics 7, 971 (2011); C.-E. Bardyn, M. A. Baranov, C. V. Kraus, E. Rico, A. Imamoglu, P. Zoller and S. Diehl, New J. Phys. 15085001 (2013).

[22] F. Caruso, S. F. Huelga, and M. B. Plenio, Phys. Rev. Lett. 105, 190501 (2010); A. W. Chin, A. Datta, F. Caruso, S. F. Huelga and M. B. Plenio, New J. Phys 12, 065002 (2010).

[23] J. Wu, R. J. Silbey, and J. Cao, Phys.Rev.Lett. 110, $200402(2013)$.

[24] J. M. Moix, M. Khasin, J. Cao, New J. Phys. 15, 085010 (2013).

[25] A. Rai and G. S. Agarwal Phys. Rev. A 79, 053849 (2009).

[26] R. Morandotti, U. Peschel, J. S. Aitchison, H. S. Eisen- berg and Y. Silberberg, Phys. Rev. Lett. 83, 4756 (1999).

[27] T. Pertsch, P. Dannberg, W. Elflein, A. Brauer, and F. Lederer, Phys. Rev. Lett. 83, 4752 (1999).

[28] K. Shandarova, C. E. Ruter, D. Kip, K. G. Makris, D. N. Christodoulides, O. Peleg and M. Segev, Phys. Rev. Lett. 102, 123905 (2009).

[29] K. Fang, Z. Yu and S. Fan, Nature Photonics 6, 782 (2012).

[30] Notice that diagonal terms in the Hamiltonian (1) do not change equations for diagonal elements of the density matrix, and do not lead to coupling between diagonal and off-diagonal elements. However, we retain this terms, firstly, for making obvious an existence of entangled stationary state, and, secondly, to make equation for coherences between zero and single excitation subspace look like a well-known 1D equation for a classical random walk (discretized heat-transfer equation), which makes obvious preservation of the sum of coherences.

[31] H. P. Breuer and F. Petruccione, The Theory of Open Quantum Systems (Oxford University Press, Oxford, 2002).

[32] D. Mogilevtsev, G. Ya. Slepyan, E. Garusov, S. Kilin, N. Korolkova, New J. Phys. 17, 043065 (2015).

[33] R. H. Dicke, Phys. Rev. 93, 99 (1954).

[34] S. Goldstein, J. L. Lebowitz, R. Tumulka, and N. Zanghi, Phys. Rev. Lett. 96, 050403 (2006).

[35] S. Popescu, A. J. Short, and A.Winter, Nature Physics 2, 754 (2006).

[36] M. P. Muller, E. Adlam, Ll. Masanes, and N. Wiebe, Commun. Math. Phys. 340(2), 499-561 (2015).

[37] Lian-Ao Wu and D. Segal, Phys. Rev. E 77, 060101(R) (2008).

[38] R. E. Peierls, Quantum Theory of Solids, (Clarendon, Oxford, 2001).

[39] S. Mukherjee, A. Spracklen, D. Choudhury, N. Goldman, P.Ohberg, E. Andersson, and R. R. Thomson, Phys. Rev. Lett. 114, 245504 (2015).

[40] R. A. Vicencio, C. Cantillano, L. Morales-Inostroza, B. Real, Cr. Mejia-Cortes, S. Weimann, A. Szameit, and M. I. Molina, Phys. Rev. Lett. 114, 245503 (2015).

[41] R. Paschotta, H. R. Telle, and U. Keller, Noise of Solid State Lasers, in Solid-State Lasers and Applications (ed. A. Sennaroglu), CRC Press, Boca Raton, FL (2007).

[42] G. Gordon and G. Kurizki, Phys.Rev.Lett. 97, 110503 (2006); ibid Phys. Rev. A 76, 042310 (2007).

[43] J. E. Mooij, T. P. Orlando, L. S. Levitov, L. Tian , C. H. van der Wal and S. Lloyd, Science 285, 1036 (1999).

[44] A. O. Lyakhov, C. Bruder, Phys. Rev. B 74, 235303 (2006).

[45] J. Cai, A. Retzker, F. Jelezko and M. B. Plenio, Nature Physics 9, 168173 (2013). 